\title{
Case Report and Review
}

\section{Idiopathic infratentorial superficial siderosis of the central nervous system: case report and review of literature}

\author{
Andrea Stabile ${ }^{a}$, Vincenzo Di Lazzaro ${ }^{b}$, Cesare Colosimo $^{c}$, Fabrizio Piazza $^{d}$, \\ Carlo Ferrarese $^{a, d}$, Jacopo C. DiFrancesco ${ }^{a, d, *}$ \\ ${ }^{a}$ Department of Neurology, ASST San Gerardo Hospital, Monza, Italy \\ ${ }^{\mathrm{b}}$ Unit of Neurology, Neurophysiology, Neurobiology, Department of Medicine, University Campus Bio-Medico, Rome, \\ Italy \\ ${ }^{\mathrm{c}}$ Radiology and Neuroradiology Unit, Diagnostic Imaging Area, Fondazione Policlinico Universitario A. Gemelli, \\ Institute of Radiology, School of Medicine, Catholic University, Rome, Italy \\ ${ }^{\mathrm{d}}$ Milan Center for Neuroscience, School of Medicine and Surgery, University of Milano-Bicocca, Monza, Italy
}

\section{A R T I C L E I N F O}

Article history:

Received 11 September 2017

Accepted 18 October 2017

Available online 27 October 2017

Keywords:

Superficial siderosis

Cerebral amyloid angiopathy

Hemosiderin

Brain Magnetic Resonance Imaging

(MRI)

Susceptibility weighted imaging

\begin{abstract}
A B S T R A C T
The superficial siderosis (SS) of the central nervous system (CNS) is a rare condition characterized by a wide range of neurological manifestations directly linked to an acquired iron-mediated neurodegeneration. First described more than 100 years ago, only recently SS has been divided into two distinct entities, according to the distribution of iron deposition in the CNS: cortical superficial siderosis (cSS) and infratentorial superficial siderosis (iSS). Here we describe an adult case of iSS, with detailed clinical and radiological features. Moreover, we extensively review the literature of SS, particularly focusing on the pathogenesis, clinical-radiological classification, diagnostic algorithm and treatment options of this rare condition.
\end{abstract}

@ 2017 Published by Elsevier Sp. z o.o. on behalf of Polish Neurological Society.

\section{Case report}

A 45-year-old Caucasian man presented with an 8-year history of slowly progressive gait difficulties, tinnitus and emotional liability. The patient also reported pharmacoresistant migraine since the age of 23, with gradual increase in intensity and number of attacks over the last few years. At the age of 27, he experienced a transient episode of acute headache and diplopia without papilledema, treated with oral corticosteroid therapy with gradual resolution of the symptoms. At that time, brain computed tomography (CT) scan, brain and spinal cord magnetic resonance imaging (MRI) were unremarkable.

\footnotetext{
* Corresponding author at: Department of Neurology, San Gerardo Hospital, School of Medicine and Surgery, University of Milano-Bicocca, Via Pergolesi, 33, 20052 Monza (MB), Italy.

E-mail addresses: jacopo.difrancesco@unimib.it, jacopo.difrancesco@gmail.com (J.C. DiFrancesco). https://doi.org/10.1016/j.pjnns.2017.10.006 0028-3843/@ 2017 Published by Elsevier Sp. z o.o. on behalf of Polish Neurological Society.
} 


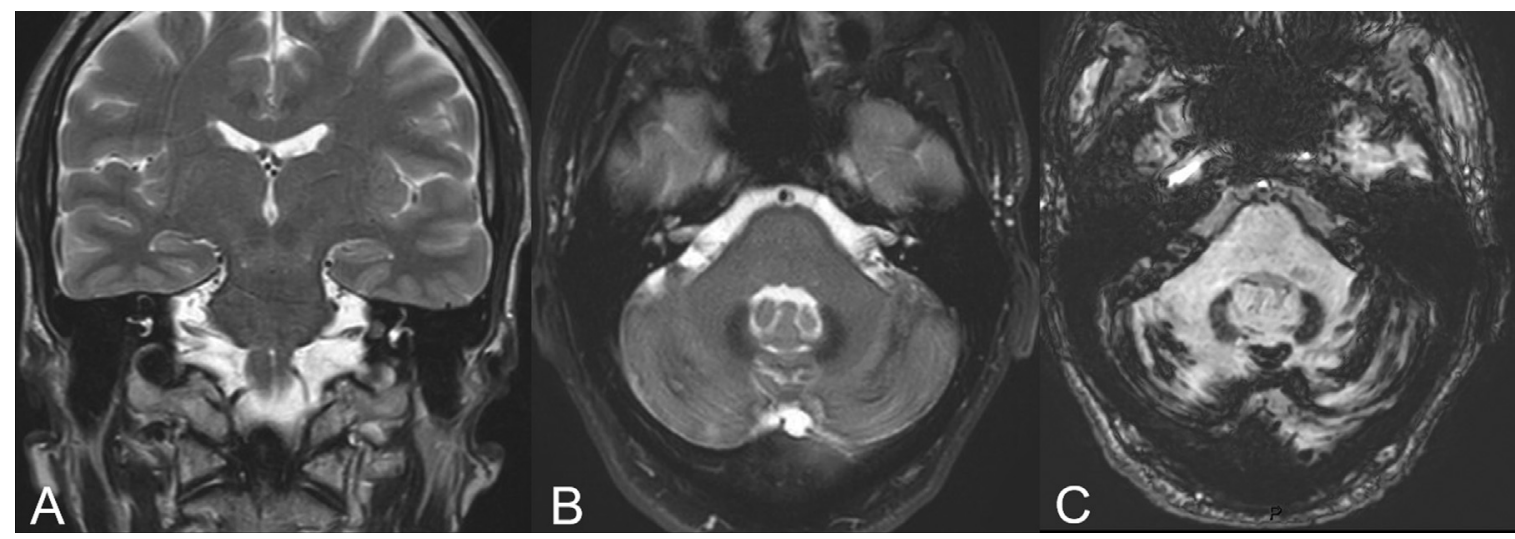

Fig. 1 - Brain MRI showing the principal neuroradiological features of iSS. (A) Coronal FSE T2-weighted image. (B) Axial fat-saturated FSE T2-weighted image. (C) Axial SWI T2*-weighted image. On FSE T2-WI (A and B) only a faint rim of hypointensity is seen on the brainstem and cerebellar surface. On T2*WI obtained by susceptibility weighted imaging sequence (SWI) there is a prominent evidence of paramagnetic deposition (hemosiderin) along the surface of the brainstem and cerebellum, as well as on the cisternal course of cranial nerves.

At the age of 44 , a $1.5 \mathrm{~T}$ brain MRI, including T2* gradient echo (GRE) and susceptibility-weighted imaging (SWI) sequences, revealed a prominent hypointensity mainly along the surface of the infratentorial structures, particularly on the surface of the brainstem, the intracranial part of facial and vestibulocochlear nerves bilaterally and the cerebellum (paravermis cerebellar cortex and dentate nuclei), associated with atrophy of the cerebellar vermis (Fig. 1). No intraparenchymal hemorrhagic lesions, nor contrast enhancement were reported. No signs of intracranial hypertension nor pseudotumor cerebri were identified. Spinal cord MRI did not reveal any relevant disease, such as neoplasms, vascular malformations and dural abnormalities, in particular dural fistula or intraspinal fluid-filled collection.

The patient was then admitted to our Department for further diagnostic investigations. There was no evidence of arterial hypertension nor injury of the brain and spinal cord. Familiar history was unremarkable for neurological diseases. Neurological examination revealed horizontal bilateral nystagmus, minor increase in muscle tone at the lower limb bilaterally and hypoesthesia/dysesthesia at the left lower limb. Deep tendon reflexes were diffusely brisk and Babinski's sign was slightly positive bilaterally. Slight oscillations in Romberg were noted and gait was wide-based and unsteady, without marked ataxic features. The patient did not report neck stiffness or hearing loss. There was no abnormality in his coordination and no evidence of cognitive impairment. Routine laboratory tests were unremarkable. Cerebral and cervical digital subtraction angiography (DSA) resulted negative for aneurysms, other vascular malformations or any cause of hemosiderin deposition. The patients refused the execution of the lumbar puncture. The electromyography excluded the presence of polyneuropathy. Rectal mucosa biopsy excluded systemic amyloidosis.

Based on clinical features and neuroimaging findings, no major cause of occult bleeding in the CNS was identified and diagnosis of idiopathic isS of the CNS was performed.

\section{Review of literature}

The SS of the CNS is an acquired neurological disorder resulting from hemosiderin deposition in the subpial layers of the brain, cranial nerves and spinal cord producing a progressive white matter and neuronal damage [1]. Hamill defined SS as "melanosis of the brain, cord and meninges" in the first report in literature [2]. Although previously defined as a rare condition, thanks to the development of specific MRI sequences for the detection of hemosiderin in the CNS, SS has been increasingly recognized in the recent past.

Even if the pathogenic mechanisms are not known yet, SS is supposed to result from a subtle, low-volume, enduring or repetitive, leakage of blood ("minor bleeding") into the subarachnoid space [3]. Other important phases contribute to the clinico-radiological expression of SS: blood dissemination by the cerebrospinal fluid (CSF), hemolysis and entrance of heme into the exposed tissue, conversion of heme in free iron, ferritin and hemosiderin and, in the end, direct tissue damage. Only CNS tissues convert the heme in CSF to hemosiderin. In response to the exposure to the heme, Bergmann glia and microglia synthetize hemoxygenase-1 (HO-1) and ferritin. HO1 breaks down the heme into free iron and biliverdin, while ferritin binds free iron to produce hemosiderin, the primary cause of the "dark rim", corresponding to the radiological hallmark of SS [4,5]. Chronic or intermittent red blood cells' extravasation in the subarachnoid space overwhelms toxic iron sequestering of glial cells, leading to iron accumulation with oxidative damage, membrane dysfunction and consequent neurodegeneration [1]. Usually, a period of many years is between the bleeding and the onset of the clinicoradiological signs of SS [5]. In the CNS, the posterior fossa seems to be mainly affected by the iron deposition because of the abundance of microglia and Bergmann glia, in particular in the cerebellum and vestibulocochlear nerves [6]. Furthermore, secondary atrophy of the cerebellar convexities and superior 
vermis are frequent consequences of iron accumulation, in relation to the pattern of CSF flow [7]. In this scenario, basal frontal lobes, mesial temporal lobes, optic nerves, olfactory tracts, spinal cord and filum terminalis are also particularly vulnerable to the toxic insult of hemosiderin deposition [8].

According to the different radiological features, clinical presentation and etiology, two principal patterns of SS are recognizable: cortical superficial siderosis (cSS) and infratentorial superficial siderosis (iSS) [5]. cSS is restricted to the supratentorial structures of the CNS, in particular, the convexities of the cerebral hemispheres $[9,10]$. Probably, cSS follows from residues originated by subacute and chronic bleeding, mainly from subarachnoid hemorrhage (SAH) $[8,10]$. This differentiates cSS from acute convexity SAH, which rather shows blood products in hyperacute or acute stages [4]. Based on the extent, cSS is classified as focal, if three or fewer sulci are affected, or disseminated, if at least four sulci are involved. Among the possible etiologies of cSS, prior or recent head trauma should be excluded first; then a non-traumatic etiology should be investigated [4]. Cerebral amyloid angiopathy (CAA), a cerebral small vessel disorder typical of the old age [11], was identified as the most frequent non-traumatic cause of cSS [12]. CAA is characterized by progressive amyloid- $\beta$ accumulation within cortical and leptomeningeal vessel walls [13], causing both hemorrhagic and ischemic brain injury $[13,14]$. It is associated with spontaneous symptomatic lobar intracerebral hemorrhage (ICH) (>5 mm in diameter) [13], and other distinctive MRI red flags represented by multiple lobar microbleeds ( $<5 \mathrm{~mm}$ in diameter) [15], white matter hyperintensity (leukoaraiosis), cSS [10] and cerebral microinfarcts [14]. There are several mechanisms by which CAA can cause cSS: the first is due to repeated episodes of acute SAH from fragile leptomeningeal or superficial cortical CAA-affected vessels; a second mechanism relates to the leakage or expansion of a lobar ICH or superficial lobar microbleeds into the subarachnoid space; the last one consists in hemorrhagic transformation of small cortical infarcts [4]. Linn et al. established the primary subarachnoid bleeding as the most likely mechanism underling the pathogenesis of cSS [10]. Other conditions associated with cSS include: reversible cerebral vasoconstriction syndrome (RCVS), primary angiitis of the CNS (PACNS) [16], CAA-related inflammation (CAA-ri) [17-20], hyperperfusion syndrome after revascularization (carotid stenting or carotid endoarterectomy), cortical vein occlusions, posterior reversible leukoencephalopathy syndrome (PRES), parent vessel stenosis, coagulopathy, drug use, cavernoma and brain abscesses [21]. Clinically cSS can present with headache, focal neurological deficits, transient focal neurological episodes (also known as "amyloid spells" [22], which can mimic transient ischemic attacks), and cognitive impairment [23], in particular when a disseminated form of siderosis is detected [12].

iSS is a recently recognized clinico-radiological entity, defined by the siderosis involving bilaterally the surface of at least two of the regions: brainstem (including midbrain, pons, medulla), cerebellum (including cerebellar folia, cerebellar peduncles, vermis), spinal cord or craniocervical junction, with or without supratentorial spreading [5]. Typically, iss is present in the crests of cerebellar folia, superior vermis, vestibulocochlear nerves, quadrigeminal plate and basal cerebral surface, but it may also engage interhemispheric fissure, Sylvian fissure, cortical sulci and the pial surface of the cord. There is a wide range of hypothesized pathogenetic causes of iSS, including SAH from intracranial aneurysms, arteriovenous malformations, tumors, CAA and spinal epidural CSF collections [24,25]. On the basis of the presumed etiology, Wilson et al. also identified two distinct groups of iss termed "classical" or type 1 iss and "secondary" or type 2 iss. Classical/type 1 iss occurs when there is no obvious, spontaneous or traumatic, CNS bleeding that could account for siderosis. It is mainly linked to dural abnormalities (mainly pseudomeningocele, extra-arachnoid CSF collection, dural ectasia), but CNS tumors, previous spinal trauma or previous neurosurgery, nerve root avulsion, brachial plexus injury and rarer diseases inducing injury or distortion of the dura (such as neurofibromatosis 1, Marfan syndrome, ankylosing spondylosis) have also been reported. In this case, the bleeding source has low pressure, probably originating from microvascular or venous blood leakage and most likely arising in the spine or in the posterior fossa at the site of a dural defect $[5,26]$. Indeed, type 1 iss is clinically characterized by the slowly progressive onset of at least one symptom of the typical triad: sensorineural hypoacusia, cerebellar ataxia, myelopathy (with pyramidal signs). Other frequent neurological manifestations are bladder/bowel defects (mainly sphincter dysfunction), swallowing deficits and anosmia [5]. Other neurological manifestations mentioned in cases already reported in literature before the classification proposed by Wilson et al. and ascribable to the classical form of iSS, are represented by seizures, memory impairment leading to dementia [27], anisocoria and sensory signs. Less frequent features include ageusia, headache, back pain [24], cranial nerve palsies and lower motoneuron signs. Secondary or type 2 iss occurs when there is an evident single hemorrhage (spontaneous ICH, aneurysmal SAH, intraventricular hemorrhage, surgical trauma and bilateral subdural hemorrhages) that, based on its spatial distribution and timing, is thought to be the cause of siderosis. In this case, a significant volume of blood rapidly accumulates (highpressure arterial leak) and is deposited in the subpial layers of the CNS tissue, mainly around the site of bleeding [5]. Therefore, clinical correlates are characterized by an acute onset [5], the location of hemosiderin deposition corresponds to the symptomatic areas of the CNS and its amount is proportional to the disease burden.

Before the advent of MRI, SS of the CNS could only be diagnosed by clinical findings and CSF analysis, with a confirmation possible only post-mortem by histopathological examination of the brain and spine [28]. With the increasing use of neuroimaging and the development of the ironsensitive sequences, MRI of the brain and spinal cord has become the cornerstone for the diagnosis of SS. MRI appearance of SS results from paramagnetic blood byproducts (hemosiderin), which cause local magnetic field inhomogeneity producing a signal loss on T2*-GRE and SWI sequences with a characteristic linear/gyral pattern beneath all CNS surfaces in contact with the CSF; in particular the availability of SWI resulted in a superior sensitivity in detecting siderosis and other paramagnetic effects [29]. Ependymal hypointensity may also occur in severe forms of siderosis. This could be related to the reflux of hemorrhagic 
CSF into the ventricles, even if the bleeding source is located outside the ventricular system. Other investigations aimed to locate a possible bleeding source in the CNS include computed tomographic angiography, magnetic resonance angiography, intra-arterial DSA of the brain or the spine, CT myelography [8] and surgical exploration. As reported in the flow-chart for isS diagnostic process, CSF analysis still preserves a role, mainly for red blood cells count, ferritin, oxyhemoglobin and bilirubin evaluation [5]. CSF is usually altered in up to $75 \%$ of cases of SS with abundant red cells and/ or xanthochromia, elevated protein and ferritin levels. According to this diagnostic pathway, in Wilson's population a cause of the SS was found in the $94 \%$ of patients with classical iss, while only $6 \%$ remained without an underlying etiology [5]. This rate of diagnostic accuracy is significantly higher compared to the $35 \%$ of unexplained cases of SS reported previously in literature and termed "idiopathic" [1].

The natural history and clinical evolution of SS are poorly understood. The identification and resolution of the bleeding source do not bring to a prompt clinical recovery or radiological reversal of SS in the majority of cases [30]. Several therapeutic approaches have been proposed, however with a mild benefit. These can be divided into two groups: the first, aimed at ablating the source of bleeding (essentially surgical approaches) and the second, aimed at reverting iron deposition (principally pharmacological approaches). Surgical treatment of SS has the goal of interrupting the blood leakage into subarachnoid space and consists in repairing the structural CNS abnormality, such as nerve root avulsion, arteriovenous malformation, dural defect, meningocele, vessels and possible membranes associated with pseudomeningocele [5,27]. However, despite some cases report a clinical improvement after surgical procedures, most of the authors agree that the disease progresses secondarily, even without an evident source of bleeding, with an unknown mechanism, likely related to the established overload of iron in the CNS [27]. The pharmacological treatment of SS includes different strategies, mainly aimed at iron chelation. During the past years, different medications have been experimented: desferrioxamine [1]; trientine and, eventually, deferiprone [31-33]. Koeppen and Dickson reported a dramatic reduction in CNS hemosiderin deposition in an animal model of SS using tin-protoporphyrin [34], an inhibitor of HO-1, but no randomized trial was conducted in humans. Although the absence of diseasemodifying treatments in classical iss, there is a preliminary evidence for the safety and tolerability of deferiprone $[31,32,35]$, which is the only iron chelator crossing the blood-brain barrier and binding hemosiderin in the CNS [36]. Deferiprone was approved for the treatment of thalassemia with iron overload secondary to blood transfusion in the USA in October 2011 [33]. Levy et al. reported the first case of SS treated with deferiprone in literature, showing MRI reduction in hemosiderin deposition in the brain [31]. A subsequent small trial on 10 participants with SS confirmed the positive trend on the MRI of four patients treated for 90 days [32]. However, no clinical improvement was observed and further studies are needed to better evaluate the efficacy of deferiprone [32]. Another possible pharmacological approach is represented by corticosteroids. These were used with clinical benefit in a single case of SS associated with anti-Ri autoimmune disease [37], while resulted ineffective in others $[38,39]$.

\section{Discussion}

SS is a neurological entity still barely diagnosed, without a specific treatment available. Brain MRI with iron-sensitive sequences represents the first-line approach to recognize and characterize SS of the CNS. Standardized radiological techniques are important to classify the different patterns of presentation of SS. Wilson et al. proposed novel diagnostic criteria, which are inspirational for future clinical research, with the final aim to identify a potential treatment. Nevertheless, besides accurate MRI studies, in the diagnostic flow-chart, it is important to keep in mind all the investigations useful for the identification of the site of bleeding.

The patient here reported showed neurological signs highly suggestive of classical iss, mainly a mild spasticity at lower limbs with imbalance and gait abnormality, associated with the radiological evidence of hemosiderin deposition in the posterior fossa. Nonetheless, he also presented with other less canonical manifestations, such as migraine, tinnitus and lateralized hypoesthesia/dysesthesia at the lower limbs, which conditioned his long diagnostic history. Notably, although we were not able to perform a lumbar puncture, his radiological picture is indicative of a form of idiopathic iSS, as supported by brain and spinal cord MRI and cerebral and cervical DSA, all resulted negative for any possible cause of bleeding.

Since the neurological damage is related to the time of exposure to iron toxic effect, a rapid diagnosis of SS and a prompt comprehension of its etiological mechanism would be desirable in order to treat the underlying condition, mostly with a surgical approach. In those cases remained without a documented bleeding source after appropriate diagnostic investigations, the development of new pharmacological treatments would be crucial to facilitate the disposal of the dangerous iron load and to provide the damaged nervous structures with the substrate necessary to promote a functional improvement.

Conflict of interest

None declared.

\section{Acknowledgement and financial support}

None declared.

REFERENCES

[1] Levy M, Turtzo C, Llinas RH. Superficial siderosis: a case report and review of the literature. Nat Clin Pract Neurol 2007;3:54-8. quiz 9.

[2] Hamill RC. Report of a case of melanosis of the brain, cord and meninges. J Nerv Ment Dis 1908;35:594. 
[3] Iwanowski L, Olszewski J. The effects of subarachnoid injections of iron-containing substances on the central nervous system. J Neuropathol Exp Neurol 1960;19:433-48.

[4] Charidimou A, Linn J, Vernooij MW, Opherk C, Akoudad S, Baron JC, et al. Cortical superficial siderosis: detection and clinical significance in cerebral amyloid angiopathy and related conditions. Brain 2015;138:2126-39.

[5] Wilson D, Chatterjee F, Farmer SF, Rudge P, McCarron MO, Cowley P, et al. Infratentorial superficial siderosis: classification, diagnostic criteria, and rational investigation pathway. Ann Neurol 2017;81:333-43.

[6] Koeppen AH, Dickson AC, Chu RC, Thach RE. The pathogenesis of superficial siderosis of the central nervous system. Ann Neurol 1993;34:646-53.

[7] Bracchi M, Savoiardo M, Triulzi F, Daniele D, Grisoli M, Bradac GB, et al. Superficial siderosis of the CNS: MR diagnosis and clinical findings. AJNR Am J Neuroradiol 1993;14:227-36.

[8] Kumar N. Neuroimaging in superficial siderosis: an indepth look. AJNR Am J Neuroradiol 2010;31:5-14.

[9] Charidimou A, Peeters AP, Jäger R, Fox Z, Vandermeeren Y, Laloux $\mathrm{P}$, et al. Cortical superficial siderosis and intracerebral hemorrhage risk in cerebral amyloid angiopathy. Neurology 2013;81:1666-73.

[10] Linn J, Herms J, Dichgans M, Brückmann H, Fesl G, Freilinger $\mathrm{T}$, et al. Subarachnoid hemosiderosis and superficial cortical hemosiderosis in cerebral amyloid angiopathy. AJNR Am J Neuroradiol 2008;29:184-6.

[11] Viswanathan A, Greenberg SM. Cerebral amyloid angiopathy in the elderly. Ann Neurol 2011;70:871-80.

[12] Lummel N, Wollenweber FA, Demaerel P, Bochmann K, Malik R, Opherk C, et al. Clinical spectrum, underlying etiologies and radiological characteristics of cortical superficial siderosis. J Neurol 2015;262:1455-62.

[13] Charidimou A, Gang Q, Werring DJ. Sporadic cerebral amyloid angiopathy revisited: recent insights into pathophysiology and clinical spectrum. J Neurol Neurosurg Psychiatry 2012;83:124-37.

[14] Gregoire SM, Charidimou A, Gadapa N, Dolan E, Antoun N, Peeters A, et al. Acute ischaemic brain lesions in intracerebral haemorrhage: multicentre cross-sectional magnetic resonance imaging study. Brain 2011;134:2376-86.

[15] Greenberg SM, Vernooij MW, Cordonnier C, Viswanathan A, Al-Shahi Salman R, Warach S, et al. Cerebral microbleeds: a guide to detection and interpretation. Lancet Neurol 2009;8:165-74.

[16] Cuvinciuc V, Viguier A, Calviere L, Raposo N, Larrue V, Cognard C, et al. Isolated acute nontraumatic cortical subarachnoid hemorrhage. AJNR Am J Neuroradiol 2010;31:1355-62.

[17] Savoiardo M, Erbetta A, Di Francesco JC, Brioschi M, Silani V, Falini A, et al. Cerebral amyloid angiopathy-related inflammation: an emerging disease. Neuroradiol J 2011;24:253-7.

[18] Auriel E, Charidimou A, Gurol ME, Ni J, Van Etten ES, Martinez-Ramirez S, et al. Validation of clinicoradiological criteria for the diagnosis of cerebral amyloid angiopathyrelated inflammation. JAMA Neurol 2016;73:197-202.

[19] DiFrancesco JC, Brioschi M, Brighina L, Ruffmann C, Saracchi E, Costantino G, et al. Anti-A $\beta$ autoantibodies in the CSF of a patient with CAA-related inflammation: a case report. Neurology 2011;76:842-4.

[20] Piazza F, Greenberg SM, Savoiardo M, Gardinetti M, Chiapparini L, Raicher I, et al. Anti-amyloid $\beta$ autoantibodies in cerebral amyloid angiopathy-related inflammation: implications for amyloid-modifying therapies. Ann Neurol 2013;73:449-58.

[21] Khurram A, Kleinig T, Leyden J. Clinical associations and causes of convexity subarachnoid hemorrhage. Stroke 2014;45:1151-3.

[22] Charidimou A, Law R, Werring DJ. Amyloid "spells" trouble. Lancet 2012;380:1620.

[23] Wollenweber FA, Buerger K, Mueller C, Ertl-Wagner B, Malik R, Dichgans M, et al. Prevalence of cortical superficial siderosis in patients with cognitive impairment. J Neurol 2014;261:277-82.

[24] Kumar N, Miller GM, Piepgras DG, Mokri B. A unifying hypothesis for a patient with superficial siderosis, lowpressure headache, intraspinal cyst, back pain, and prominent vascularity. J Neurosurg 2010;113:97-101.

[25] Hoxworth JM, Patel AC, Bosch EP, Nelson KD. Localization of a rapid CSF leak with digital subtraction myelography. AJNR Am J Neuroradiol 2009;30:516-9.

[26] Gaudino S, Colantonio R, Schiarelli C, Martucci M, Calandrelli R, Botto A, et al. Postoperative MR imaging of spontaneous transdural spinal cord herniation: expected findings and complications. AJNR Am J Neuroradiol 2016;37:558-64.

[27] Posti JP, Juvela S, Parkkola R, Roine S. Three cases of superficial siderosis of the central nervous system and review of the literature. Acta Neurochir (Wien) 2011;153:2067-73.

[28] Hughes JT, Oppenheimer DR. Superficial siderosis of the central nervous system. A report on nine cases with autopsy. Acta Neuropathol 1969;13:56-74.

[29] Greenberg SM, Finklestein SP, Schaefer PW. Petechial hemorrhages accompanying lobar hemorrhage: detection by gradient-echo MRI. Neurology 1996;46:1751-4.

[30] Koeppen AH, Michael SC, Li D, Chen Z, Cusack MJ, Gibson WM, et al. The pathology of superficial siderosis of the central nervous system. Acta Neuropathol 2008;116:371-82.

[31] Levy M, Llinas RH. Deferiprone reduces hemosiderin deposits in the brain of a patient with superficial siderosis. AJNR Am J Neuroradiol 2011;32:E1-2.

[32] Levy M, Llinas R. Pilot safety trial of deferiprone in 10 subjects with superficial siderosis. Stroke 2012;43:120-4.

[33] Cummins G, Crundwell G, Baguley D, Lennox G. Treatment of superficial siderosis with iron chelation therapy. BMJ Case Rep 2013;2013.

[34] Koeppen AH, Dickson AC. Tin-protoporphyrin prevents experimental superficial siderosis in rabbits. J Neuropathol Exp Neurol 2002;61:689-701.

[35] Levy M, Llinas RH. Update on a patient with superficial siderosis on deferiprone. AJNR Am J Neuroradiol 2012;33: E99-100.

[36] Fredenburg AM, Sethi RK, Allen DD, Yokel RA. The pharmacokinetics and blood-brain barrier permeation of the chelators 1,2 dimethly-, 1,2 diethyl-, and 1-[ethan-1'ol]2-methyl-3-hydroxypyridin-4-one in the rat. Toxicology 1996;108:191-9.

[37] Angstwurm K, Schielke E, Zimmer C, Kivelitz D, Weber JR. Superficial siderosis of the central nervous system: response to steroid therapy. J Neurol 2002;249:1223-5.

[38] Scheid R, Frisch S, Schroeter ML. Superficial siderosis of the central nervous system - treatment with steroids? J Clin Pharm Ther 2009;34:603-5.

[39] Leussink VI, Flachenecker P, Brechtelsbauer D, Bendszus M, Sliwka U, Gold R, et al. Superficial siderosis of the central nervous system: pathogenetic heterogeneity and therapeutic approaches. Acta Neurol Scand 2003;107:54-61. 